Paper

\title{
The double-assignment method for the exponential chaotic tabu search in quadratic assignment problems
}

\author{
Kazuaki Shibata $^{1 a)}$, Yoshihiko Horio ${ }^{1 b)}$, and Kazuyuki Aihara ${ }^{2 c)}$ \\ ${ }^{1}$ Graduate of School of Engineering, Tokyo Denki University \\ 2-2 Kanda-Nishiki-cho, Chiyoda-ku, Tokyo 101-8457, Japan \\ ${ }^{2}$ Institute of Industrial Science, The University of Tokyo \\ 4-6-1 Komaba, Meguro-ku, Tokyo 153-8505, Japan \\ a) $k_{-}$shibata@eee.dendai.ac.jp \\ b) horio@eee.dendai.ac.jp \\ c)aihara@sat.t.u-tokyo.ac.jp
}

Received February 15, 2011; Revised June 20, 2011; Published October 1, 2011

\begin{abstract}
The quadratic assignment problem (QAP) is one of the $\mathcal{N} \mathcal{P}$-hard combinatorial optimization problems. An exponential chaotic tabu search using a 2-opt algorithm driven by chaotic neuro-dynamics has been proposed as one heuristic method for solving QAPs. In this paper we first propose a new local search, the double-assignment method, suitable for the exponential chaotic tabu search, which adopts features of the Lin-Kernighan algorithm. We then introduce chaotic neuro-dynamics into the double-assignment method to propose a novel exponential chaotic tabu search. We further improve the proposed exponential chaotic tabu search with the double-assignment method by enhancing the effect of chaotic neuro-dynamics.
\end{abstract}

Key Words: double-assignment method, exponential chaotic tabu search, chaotic neural network, quadratic assignment problem, Lin-Kernighan algorithm

\section{Introduction}

A great deal of effort has been made to solve combinatorial optimization problems, in particular, in the area of operations research and meta-heuristics [1]. Through those researches, various effective search algorithms to obtain approximate solutions were proposed such as the 2-opt algorithm [2], $k$-opt algorithm [3], Or-opt algorithm [4], Lin-Kernighan (LK) algorithm [5], and stem-and-cycle ejection chain algorithm [6]. In addition, a variety of meta-heuristics, e.g. simulated annealing [7], genetic algorithm, genetic hybrids [8], tabu search [9], reactive tabu search [10], robust tabu search [11] and so on, were proposed to avoid the local minimum problem.

The quadratic assignment problem (QAP) is one well-known $\mathcal{N} \mathcal{P}$-hard combinatorial optimization problem [12-16]. For QAPs, many local searches and meta-heuristics have been proposed and studied $[1,17]$. In particular, tabu search and its derivatives with the 2-opt exchanges have been used as 
effective meta-heuristic algorithms [18].

On the other hand, since the dynamical memory search was demonstrated in a dynamical associative memory with a chaotic neural network model [19-21], chaotic dynamics have been applied to combinatorial optimization problems. In the early stage of these approaches, chaotic dynamics were used as a noise source $[22,23]$. In recent studies, however, chaotic dynamics are more actively used in the following two ways: (a) Chaotic dynamics are introduced into a mutually connected neural network, and (b) a local search is controlled by chaotic neuro-dynamics. In particular, the approach (b), such as an exponential chaotic tabu search, has been shown to be very effective and practical in several combinatorial optimization problems.

The exponential chaotic tabu search, in which the 2-opt exchanges are controlled by chaotic neurodynamics, was first applied to the traveling salesman problem (TSP) [24]. The exponential chaotic tabu search, then, has been improved by combining chaotic neuro-dynamics with the Or-opt algorithm, $k$-opt algorithm, LK algorithm, and stem-and-cycle ejection chain algorithm. As a result, TSPs with the size of $10^{5}$ to $10^{7}$ can be efficiently solved through the chaotic search [25-30]. In addition to TSPs, the exponential chaotic tabu search with chaotic neuro-dynamics was also applied to QAPs [31,32], motif extraction problems [33], vehicle routing problems with time window [34], and packet routing problems [35]; furthermore, effectiveness of the exponential chaotic tabu searches was demonstrated through these problems.

Salient features of the exponential chaotic tabu search are an exponential decaying continuous tabu (exponential tabu) effect, and an efficient dynamical search in the state-space through chaotic itinerancy [36]. The exponential tabu is naturally introduced in the exponential chaotic tabu search dynamics by the refractoriness of the chaotic neuron $[19,21,31,32]$. The tabu intensity in the exponential chaotic tabu search takes continuous value, and decreases exponentially by the refractoriness of the chaotic neuron $[31,32]$. Moreover, the intensity and decay time-constant of the tabu effect can be easily controlled by the refractory parameters of the chaotic neurons [19,21].

On the other hand, chaotic itinerancy refers to universal dynamics in high-dimensional dynamical systems, showing itinerant motion among varieties of low-dimensional ordered states (quasi-attractors or attractor ruins) through high-dimensional chaos [36]. The chaotic attractors usually have fractal structures. The chaotic search dynamics search the solutions along such chaotic attractors with zero Lebesgue measure in the state space, so that the chaotic search is expected to be efficient. Moreover, the itinerant motion near the attractor ruins would make the local search deep, and at the same time, help the local search to escape from local minima.

Complexity, richness, and computational power inherent in chaotic dynamics are due to the complexity of real-numbers [37-39]. Therefore, it is mandatory to implement a chaotic dynamical system with an analog circuitry, which can handle real-numbers through continuous variables [40,41]. In addition, an analog circuit system can achieve truly and massively parallel processing as its nature. In this respect, we have constructed a general purpose chaotic neuro-computer with a switchedcapacitor analog sampled-data integrated-circuit technique [40,41]. Moreover, we have implemented an exponential chaotic tabu search based on the 2-opt exchanges into a hardware system by using switched-current analog integrated circuits [41-43]. By means of experiments, we have demonstrated high-abilities of these hardware systems in solving QAPs. Furthermore, we analyzed high-dimensional spatio-temporal chaotic neuro-dynamics, which are necessary and effective to the exponential chaotic tabu search, through spatio and temporal mutual information [41,44]. In addition, a completely parallel updating scheme of the neurons has been proposed for a quick execution of the exponential chaotic tabu search with the 2-opt algorithm. The execution time of the parallel hardware systems would be independent from the size of QAPs thanks to the truly parallel nature inherent in the analog circuitry $[44,45]$.

As mentioned above, the exponential chaotic tabu search, in which the 2-opt algorithm is controlled by chaotic neuro-dynamics, was successfully applied to QAPs showing high solving performance. In this paper, we propose a further effective exponential chaotic tabu search for QAPs with a novel local search. To this end, we first propose in Section 3 a double-assignment (DA) method as a local search for QAPs [46]. In the DA method, features of the LK algorithm for TSPs are employed. We 
then show through numerical simulations that the DA method has better performance than the 2-opt method as a local search. Secondly, in Section 4, we propose an exponential chaotic tabu search in which chaotic neuro-dynamics control the DA method. Thirdly, we modify the DA method (modified double-assignment (MDA) method) to enhance the chaotic neuro-dynamics effect. We also evaluate the MDA method as a local search. We then adopt the MDA method in the exponential chaotic tabu search. We finally compare the performance of the exponential chaotic tabu searches with the DA method, with the MDA method, and with the 2-opt exchanges.

\section{Quadratic assignment problem}

The quadratic assignment problem (QAP) $[13,14,16]$ is one of the $\mathcal{N} \mathcal{P}$-hard combinatorial optimization problems. In this section, we briefly review the QAP using the problem of plant locations of size $N$ as an example. The goal of the plant location problem is to find an assignment of plants to unique cities that minimizes the cost function in Eq. (1).

$$
F(\boldsymbol{p})=\sum_{i=1}^{N} \sum_{j=1}^{N} a_{i j} b_{p(i) p(j)}
$$

where $a_{i j}$ gives the distance between city $i$ and city $j$, and $b_{p(i) p(j)}$ gives the flow (weight) from plant $p(i)$ to plant $p(j)$. The distances between each pair of cities, and the flows between each pair of plants are given by the distance matrix $\boldsymbol{A}$, and the flow matrix $\boldsymbol{B}$, respectively, as in Eq. (2).

$$
\boldsymbol{A}=\left[\begin{array}{ccccc}
a_{11} & \cdots & a_{1 j} & \cdots & a_{1 N} \\
\vdots & \ddots & \vdots & \ddots & \vdots \\
a_{i 1} & \cdots & a_{i j} & \cdots & a_{i N} \\
\vdots & \ddots & \vdots & \ddots & \vdots \\
a_{N 1} & \cdots & a_{N j} & \cdots & a_{N N}
\end{array}\right], \quad \boldsymbol{B}=\left[\begin{array}{ccccc}
b_{11} & \cdots & b_{1 j} & \cdots & b_{1 N} \\
\vdots & \ddots & \vdots & \ddots & \vdots \\
b_{i 1} & \cdots & b_{i j} & \cdots & b_{i N} \\
\vdots & \ddots & \vdots & \ddots & \vdots \\
b_{N 1} & \cdots & b_{N j} & \cdots & b_{N N}
\end{array}\right]
$$

Furthermore, feasible solutions can be expressed by the permutation $\boldsymbol{p}$ in Eq. (3) [15].

$$
\begin{array}{rcccccccc}
\text { index }: & 1 & 2 & \ldots & i & \ldots & j & \ldots & N \\
\boldsymbol{p}: & \{p(1), & p(2), & \ldots, & p(i), & \ldots, & p(j), & \ldots & p(N)\}
\end{array}
$$

In Eq. (3), the index $i$ of $\boldsymbol{p}$ corresponds to city $i$, and the element $p(i)$ corresponds to the plant that is assigned to city $i$.

The number of feasible solutions to a size- $N$ QAP is $N$ !, and thus it is difficult to obtain the optimum solution in a reasonable time by evaluating all feasible solutions. Therefore, some heuristic methods have been proposed to quickly obtain sub-optimum solutions $[1,8-13,16-18]$. The 2 -opt exchange [47] is one of the most basic local search heuristics. It exchanges two plants if the exchange decreases $F(\boldsymbol{p})$. An example of the 2-opt exchange method for QAPs is illustrated in Fig. 1 for a size-5 QAP, where plants 2 and 5 are to be exchanged.

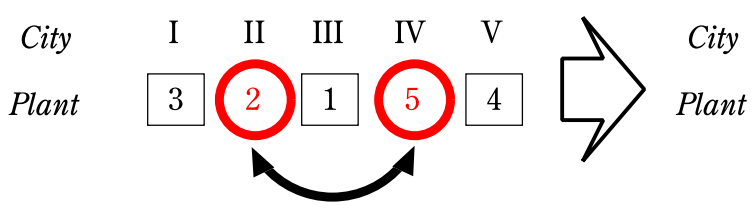

(a)

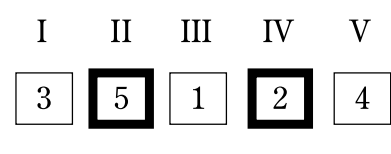

(b)

Fig. 1. An example of the 2-opt method for a size-5 QAP; (a) is the initial permutation, and (b) is the resulting permutation after the exchange of plants 2 and 5.

\section{The double-assignment method}

In this section, we propose a local search, double-assignment (DA) method, which can be available for the exponential chaotic tabu search. The DA method employs features of the LK algorithm; therefore, we start from a short review of the LK algorithm. 


\subsection{The Lin-Kernighan algorithm for TSPs}

In the TSP [1,48], which is also an $\mathcal{N} \mathcal{P}$-hard problem, the task is to find a shortest possible tour that visits all cities in a list exactly once. Many heuristics have been proposed for the TSP [1, 48], including the 2-opt method, the $k$-opt method, the Or-opt algorithm, the LK algorithm, and stem-and-cycle ejection chain algorithm $[2-6]$.

The LK algorithm [5] is known to be one of the efficient methods for solving TSPs. It swaps links like the $k$-opt method, but $k$ changes dynamically. In addition, the LK algorithm goes through infeasible solutions during the search for better tours, as shown in Fig. 2. We first choose a city $t_{1}$ at random (Fig. 2(b)). Then, we choose a city $t_{2}$ next to $t_{1}$, and trim the path $x_{1}$ between $t_{1}$ and $t_{2}$. Next, we add the route $y_{1}$ between $t_{2}$ and a city $t_{3}$, such that $y_{1}$ is shorter than $x_{1}$ (Fig. $2(\mathrm{c})$ ). After that, we choose a city $t_{4}$ next to $t_{3}$ (Fig. $2(\mathrm{~d})$ ), and trim the path $x_{2}$ between $t_{3}$ and $t_{4}$. We then evaluate the cost improvement $G^{*}$ when we temporarily add a path $T^{\prime}$ between $t_{1}$ and $t_{4}$. We next choose a city $t_{5}$ and a route $y_{2}$ between $t_{4}$ and $t_{5}$ such that $\left|x_{2}\right|+\left|x_{1}\right|>\left|y_{2}\right|+\left|y_{1}\right|$ (Fig. 2(e)), where $\left|x_{1}\right|,\left|x_{2}\right|$, $\left|y_{1}\right|$ and $\left|y_{2}\right|$ are the lengths of $x_{1}, x_{2}, y_{1}$ and $y_{2}$, respectively. If $\left(\left|x_{2}\right|+\left|x_{1}\right|\right)-\left(\left|y_{2}\right|+\left|y_{1}\right|\right)>G^{*}$, we continue to delete and add routes (Fig. 2(f)). Moreover, if $\left(\left|x_{2}\right|+\left|x_{1}\right|\right)-\left(\left|y_{2}\right|+\left|y_{1}\right|\right) \leq G^{*}$, or we cannot add $y_{2}$, we halt and update the current tour to $T^{\prime}$ (Fig. $2(\mathrm{~g})$ ).

As shown in Fig. 2, the number of link exchanges depends on the cost improvements and the lengths of the added and removed paths. Furthermore, the tours in Figs. 2(b) to 2(e) are not closed, and so are infeasible solutions. This greatly broadens the solution search space, giving the LK algorithm good performance.

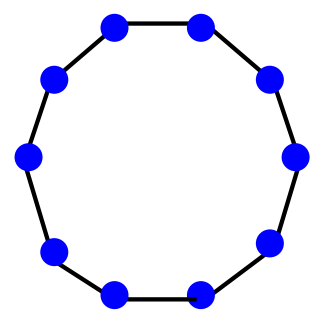

(a)

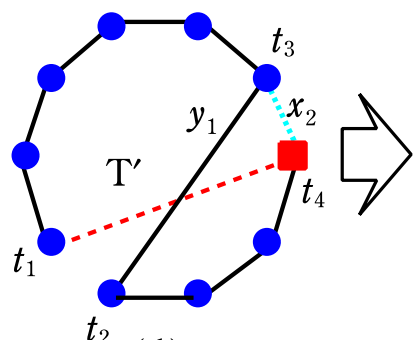

(d)

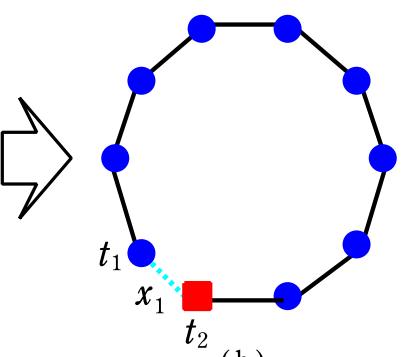

(b)

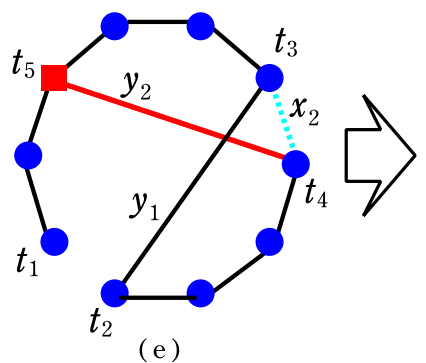

$\checkmark V$
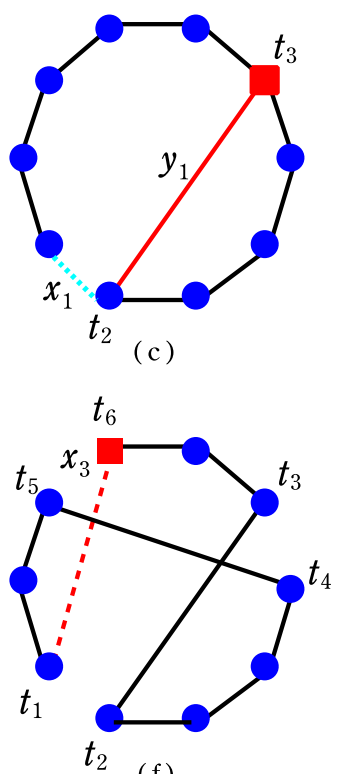

(f)

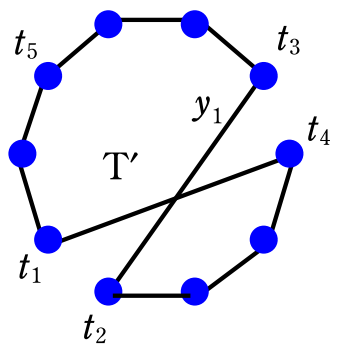

( g)

Fig. 2. An example of the Lin-Kernighan algorithm: (a) the initial tour; (b) delete the path $x_{1}$ between $t_{1}$ and $t_{2} ;$ (c) add the path $y_{1}$ between $t_{2}$ and $t_{3}$; (d) delete the path $x_{2}$ between $t_{3}$ and $t_{4}$, and evaluate the temporary path $T^{\prime}$ between $t_{1}$ and $t_{4}$; (e) add the path $y_{2}$ between $t_{4}$ and $t_{5}$; (f) continue the procedure, or $(\mathrm{g})$ halt. 
From the above, we can conclude that the LK algorithm has the following two salient features.

(F-1) The number of exchanged links changes dynamically.

(F-2) Feasible solutions are searched for through infeasible solutions.

In this respect, in this paper we propose the DA method which can be used in the exponential chaotic tabu search for QAPs, introducing feature (F-2) of the LK algorithm [46]. Namely, the DA method searches for feasible solutions through infeasible solutions.

\subsection{The double-assignment method for QAPs}

The DA method first constructs an infeasible solution in which some cities have no plants assigned, and some cities have two plants. A new feasible solution is then constructed by reassigning overassigned plants to empty cities. In the following, we illustrate the DA procedure using an example where plant $c$ is initially doubly assigned to city $d$. In Step $\mathbf{1}$ and Step $\mathbf{2}$ below, we first deliberately construct an infeasible solution, and from that construct a feasible solution in Step 3.

Step 0: Initialize the counters $m$ and $n$ as $m=0$ and $n=0$, where the parameter $m$ is a count of the number of runs of Step 1 and Step 2, and $n$ is a count of the runs of Step 3.

Step 1: Increment $m$ by one (now, $m=1$ ). Arbitrarily choose a plant $c$ and a city $d$ from the list of plants and cities, respectively, and doubly assign $c$ to $d$. Denote city $d$ as $x_{1}$, and the city to which plant $c$ was assigned as $y_{1}$.

Step 2: Increment $m$ by one $(m=m+1)$. Randomly choose a city $y_{m}$ from among the cities that have only one plant. Doubly assign the plant in city $y_{m}$ to city $x_{m}$ for which the assignment results in the minimum cost increase. Note that the list of cities from which $x_{m}$ is chosen excludes the cities $x_{1}$ to $x_{m-1}$ and $y_{1}$ to $y_{m}$. If $m+1 \leq N / 2$, repeat Step 2, otherwise proceed to Step 3.

Step 3: Increment $n$ by one $(n=n+1)$. Choose from among those plants assigned to cities $x_{1}$ to $x_{m}$ the plant that gives the minimum cost increase when it is assigned to city $y_{n}$, and assign it to $y_{n}$. Relabel the city where the chosen plant resided as $z_{n}$. If $n=m$, the algorithm ends. Otherwise, repeat Step 3.

A flow diagram of the DA method is shown in Fig. 3 for the case of a size-5 QAP. Figure 3(a) shows Step 1 in the case when "city $d "=$ "city II," and "plant $c "=$ "plant 1." There, we first doubly assign plant 1 to city II. We then denote city II as $x_{1}$, and city III as $y_{1}$. Figure 3(b) shows Step 2 when city $\mathrm{V}$ is chosen as $y_{2}$. In this step, we first calculate the cost increase when plant 4 , which was assigned to city $\mathrm{V}$, is temporarily assigned to cities I and IV, respectively, each of which has only one plant. If the assignment of plant 4 to city I gives the smallest cost increase, we doubly assign the plant 4 to city I. The first procedure of Step 3 is shown in Fig. 3(c). In this step, we evaluate the increases in the cost when plants 1, 2, 3, and 4 are each assigned to city III. If the assignment of plant 2 to city III results in the smallest cost increase, we reassign plant 2 to city III as a consequence. As a result, $z_{1}=$ "city II." Figure 3(d) shows the successive procedure of Step 3. We again evaluate the increases in the cost when the remaining plants 3 and 4 are assigned to city $\mathrm{V}$, respectively. If when plant 4 is assigned to city $\mathrm{V}$ the cost increase is smaller than the case where plant 3 was assigned, we reassign plant 4 to city V. As demonstrated in Fig. 3, the DA method constructs a new feasible solution through infeasible solutions.

The total cost improvement by one execution of double assignment can be evaluated as the sum of the cost changes after each step. Moreover, in Step 2 and Step 3, we need to calculate the increase in cost when we choose city $x_{m}$, where the plant of city $y_{m}$ is assigned, and the plant that is reassigned to city $y_{n}$. Equations (4) and (5) are used to evaluate the cost increases in Step 1 and Step 2, respectively. 


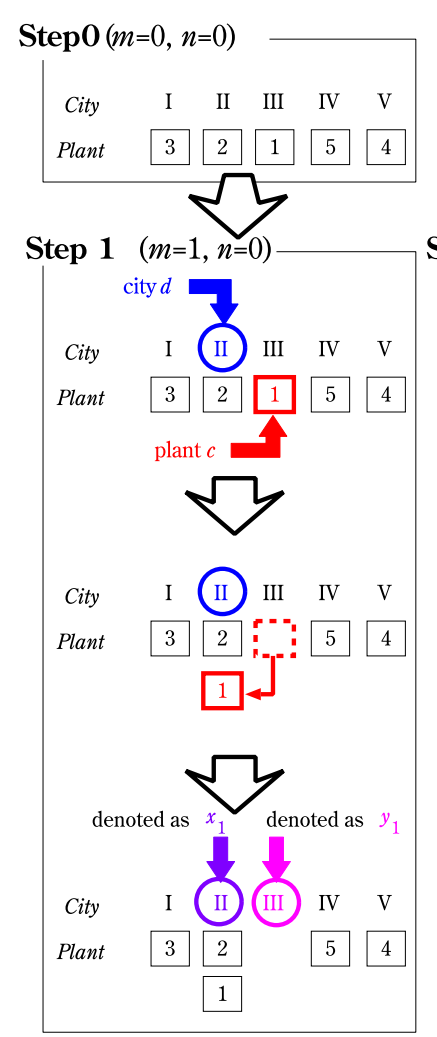

(a)

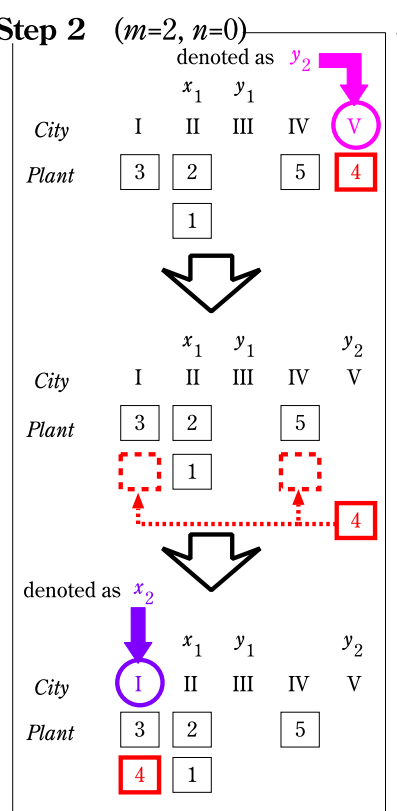

(b)

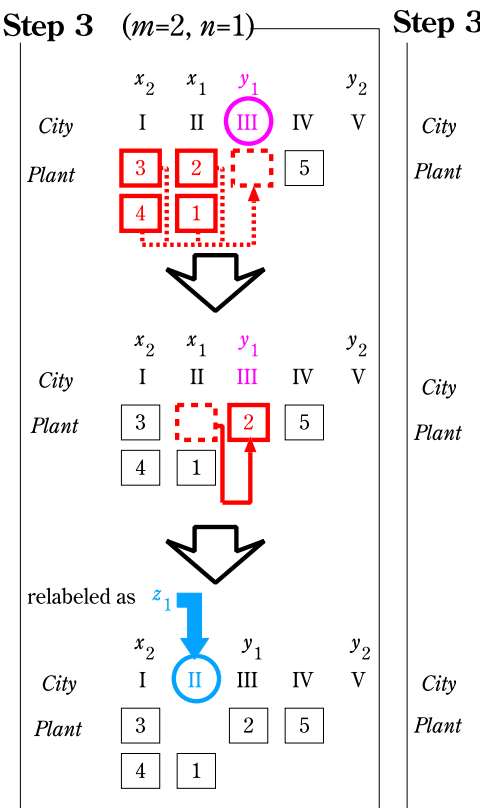

(c)

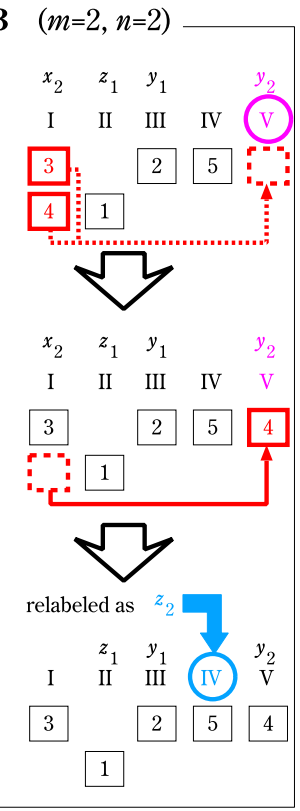

(d)

Fig. 3. An example of the double-assignment method: (a) Step 1 when plant 1 is chosen as $c$, and city II is chosen as $d$; (b) Step 2 when city V is chosen as $y_{2} ;$ (c) Step 3 when plant 2 is chosen for reassignment to city $y_{1}$; and (d)

Step 3 when plant 4 is chosen for reassignment to city $y_{2}$.

$$
\begin{aligned}
\Delta F_{i j}^{1} & =-\left(a_{i j} b_{p(i) p(j)}+a_{j i} b_{p(j) p(i)}\right)+\sum_{k=1, k \neq i, j}^{N}\left(b_{p_{1}(i) p_{1}(k)}\right)\left(a_{k j}-a_{k i}\right) \\
& +\sum_{k=1, k \neq i, j}^{N}\left(b_{p_{1}(k) p_{1}(i)}\right)\left(a_{j k}-a_{i k}\right) \\
\Delta F_{i j}^{2} & =-\left(a_{i j} b_{p(i) p(j)}+a_{j i} b_{p(j) p(i)}\right)+\sum_{k=1, k \neq i, j}^{N}\left(b_{p_{1}(i) p_{1}(k)}+b_{p_{1}(i) p_{2}(k)}\right)\left(a_{k j}-a_{k i}\right) \\
& +\sum_{k=1, k \neq i, j}^{N}\left(b_{p_{1}(k) p_{1}(i)}+b_{p_{2}(k) p_{1}(i)}\right)\left(a_{j k}-a_{i k}\right)
\end{aligned}
$$

where $\Delta F_{i j}^{1}$ is the cost increase when the plant in city $i$ is doubly assigned to city $j$ in Step $\mathbf{1}$, and $\Delta F_{i j}^{2}$ is that in Step 2. Moreover, $p(i)$ and $p(j)$ are the plants assigned to city $i$ and city $j$, respectively. In addition, $p_{1}(i)$ is the plant originally assigned to city $i$, and $p_{2}(i)$ is the plant newly assigned to city $i$. On the other hand, the cost increase in Step 3 is given by Eq. (6).

$$
\begin{aligned}
\Delta F_{i j}^{3} & =a_{l j} b_{i p(l)}+a_{j l} b_{p(l) i}+\sum_{k=1, k \neq i, j}^{N}\left(b_{i p_{1}(k)}+b_{i p_{2}(k)}\right)\left(a_{k j}-a_{k l}\right) \\
& +\sum_{k=1, k \neq i, j}^{N}\left(b_{p_{1}(k) i}+b_{p_{2}(k) i}\right)\left(a_{j k}-a_{l k}\right)
\end{aligned}
$$

where $\Delta F_{i j}^{3}$ represents the change in cost when plant $i$ is reassigned to city $j$ in Step $\mathbf{3}$. Moreover, $l$ is the city to which plant $i$ was originally assigned, and $p(j)$ and $p(l)$ are the plants assigned to city $j$ and city $l$, respectively. 


\subsection{Comparison of the 2-opt and double-assignment methods}

In this section, we compare the solving performance of the DA method and the 2-opt method as local searches through numerical simulations to confirm the effectiveness of feature (F-2) of the LK algorithm for solving QAPs. Because these methods are local searches, they would finally fall into a local minimum. Therefore, comparisons are made for the same iteration numbers defined below for each method.

In simulations of the 2-opt method, the following procedure (2opt-1 and -2) is treated as a single iteration:

2opt-1: Examine the results of all possible 2-opt exchanges to the current solution, $\pi$.

2opt-2: If $\pi_{\text {best }}$ is better than $\pi$, replace $\pi$ with $\pi_{\text {best }}$.

On the other hand, in the simulations for the DA method the following procedure (DA-1, 2, 3 and 4) is treated as a single iteration.

DA-1: Initialize each of the counters $i$ and $j$ to 1 .

DA-2: Temporarily execute the DA method, which initially assigns plant $i$ to city $j$ in Step $\mathbf{1}$, to the current solution $\pi$.

DA-3: If $j<N$, increment $j$ by one, and return to DA-2. If $j=N$ and $i<N$, increment $i$ by one. At the same time, reset $j$ to $j=1$, and go back to DA-2. If $j=N$ and $i=N$, go to DA-4.

DA-4: Take the best solution $\pi_{\text {best }}$ from all the solutions obtained from DA-2 and DA-3. If $\pi_{b e s t}$ is better than $\pi$, we replace $\pi$ by $\pi_{\text {best }}$.

In the numerical simulations, we used 5,000 iterations for each trial. We executed 30 trials with different initial conditions for each method. Table I summarizes the average gap from the optimum or known-best solution (AG), and the success rate in obtaining the optimum or known-best solution (SR), for local searches employed in the exponential chaotic tabu searches, using benchmark problems from the QAP library [16]. From the left two columns (2-opt and DA) of Table I, it is clear that the DA method results in better solutions than the 2-opt method. This result confirms that solution searches through infeasible solutions, feature (F-2) of the LK algorithm, are effective in solving QAPs.

\section{The exponential chaotic tabu search with the double-assignment method}

Although the DA method has better solving performance than the 2-opt method, like the 2-opt method it can be trapped in local minima. To solve the local minima problem, some meta-heuristic methods such as the simulated annealing [7], genetic algorithm, genetic hybrids [8], tabu search [9], reactive tabu search [10], and robust tabu search [11] have been proposed for QAPs. In addition, chaotic dynamics have been effectively utilized. In particular, the exponential chaotic tabu search for QAPs, in which the 2-opt exchanges are controlled by chaotic neuro-dynamics, is one of the effective searches which exploit high-dimensional chaotic dynamics [31,32]. In the exponential chaotic tabu search, the tabu effects exponentially decay due to the inherent refractoriness of the chaotic neurons.

The DA method is employed in the exponential chaotic tabu search in place of the 2-opt method as a local search in this section. We first briefly review the original exponential chaotic tabu search with the 2-opt method; then the chaotic neuro-dynamics is introduced into the DA method.

\subsection{The original exponential chaotic tabu search with the 2-opt method}

Candidate exchanges in the 2-opt method are determined by chaotic neuro-dynamics from a chaotic neural network in the exponential chaotic tabu search [31,32]. In the exponential chaotic tabu search, chaotic neurons in the neural network are asynchronously updated. If the $(i, j)$ th neuron fires, we assign plant $i$ to city $j$, and at the same time we assign plant $p(j)$ in city $j$ to city $q(i)$, where plant $i$ 
Table I. Comparisons among local searches used in the exponential chaotic tabu searches for QAPs. The average gap from the optimum or known-best solution $(\mathrm{AG})$, and the success rate in finding the optimum or known-best solution (SR) through 30 numerical simulation trials with 5,000 iterations for each trial, using the 2-opt method (2-opt), the double-assignment method (DA), and the modified double-assignment method (MDA). The best result and second best for each problem are shown with bold and italic numbers, respectively.

\begin{tabular}{c|rr|rr|rr}
\hline & \multicolumn{2}{|c|}{2 -opt } & \multicolumn{2}{|c|}{ DA } & \multicolumn{2}{c}{ MDA } \\
\hline Instance & AG $(\%)$ & SR $(\%)$ & AG $(\%)$ & SR $(\%)$ & AG $(\%)$ & SR (\%) \\
\hline Chr20a & 45.852 & 0 & $\mathbf{8 . 7 5 0}$ & 0 & 10.53 & 0 \\
Chr20b & 43.394 & 0 & $\mathbf{6 . 5 4 5}$ & $\mathbf{3 . 3 3}$ & 7.008 & 0 \\
Chr20c & 78.226 & 0 & $\mathbf{9 . 2 6 3}$ & $\mathbf{6 . 6 6}$ & 18.13 & 3.33 \\
Had20 & 1.174 & 3.33 & 0.499 & 3.33 & $\mathbf{0 . 1 7 5}$ & $\mathbf{5 3 . 3}$ \\
Nug20 & 3.709 & 0 & $\mathbf{1 . 0 8 9}$ & 3.33 & 1.344 & $\mathbf{6 . 6 6}$ \\
Nug30 & 2.310 & 0 & 1.595 & 0 & $\mathbf{0 . 8 9 4}$ & 0 \\
Rou20 & 5.058 & 0 & $\mathbf{1 . 4 2 6}$ & 0 & 1.467 & 0 \\
Scr20 & 8.684 & 0 & $\mathbf{1 . 6 0 9}$ & 6.66 & 1.654 & $\mathbf{1 0 . 0}$ \\
Sko42 & 3.560 & 0 & 3.125 & 0 & $\mathbf{1 . 2 0 5}$ & 0 \\
Tai20a & 6.079 & 0 & 1.772 & 0 & $\mathbf{1 . 7 5 7}$ & 0 \\
Tai30a & 3.499 & 0 & 2.624 & 0 & $\mathbf{2 . 2 0 3}$ & 0 \\
Tai40a & 5.087 & 0 & 4.921 & 0 & $\mathbf{2 . 5 3 9}$ & 0 \\
Tai20b & 16.560 & 0 & 0.325 & 40.0 & $\mathbf{0 . 3 1 7}$ & $\mathbf{5 0 . 0}$ \\
Tai30b & 16.518 & 0 & 1.243 & 0 & $\mathbf{1 . 8 0 2}$ & $\mathbf{1 3 . 3}$ \\
Tai40b & 5.087 & 0 & $\mathbf{3 . 8 2 6}$ & 0 & 4.384 & $\mathbf{1 0 . 0}$ \\
\hline
\end{tabular}

was assigned. When the $(i, j)$ th neuron fires, the $(i, j)$ th and $(p(j), q(i))$ th neurons will not fire for a while, as determined by the refractoriness of the $(i, j)$ th and $(p(j), q(i))$ th neurons, which naturally implement exponentially decaying tabu effects.

Figure 4 illustrates the exponential chaotic tabu search, in which the 2-opt exchange is controlled by the firing of chaotic neurons $[31,32]$. The figure also demonstrates the exponential tabu effect through the refractoriness of the chaotic neuron model [19,21]. In Fig. 4, the $(2,5)$ th neuron fires, so plant 2 is assigned to city $\mathrm{V}$ by a 2 -opt exchange. In addition, the $(2,5)$ th neuron and the $(4,2)$ th neuron will not fire for a while because of the exponentially decaying refractory effects.

The performance of the exponential chaotic tabu search with the 2-opt exchanges, such as the average gap from the known-best solution and the CPU-time, has been carefully and extensively compared with that of other meta-heuristic approaches, such as the stochastic simulated annealing, the robust tabu search and chaotic simulated annealing, using the instances from the QAP library in [32]. As a result, they showed that the exponential chaotic tabu search with the 2-opt exchanges is comparable to or better than the robust tabu search in terms of quality of solutions and speed. Moreover, we have confirmed their results by repeating their simulations. Therefore, we employ this original exponential chaotic tabu search with the 2-opt method as an evaluation standard for the proposed exponential chaotic tabu searches in the following simulations.

\subsection{Introducing the exponential chaotic tabu search into the double-assignment method}

As in the original exponential chaotic tabu search [31,32], we use a chaotic neural network with $N \times N$ chaotic neurons for a size- $N$ QAP in order to control the DA method. The dynamics of the $(i, j)$ th chaotic neuron in the network are given by

$$
\begin{aligned}
\zeta_{i j}(t+1) & =k_{r} \zeta_{i j}(t)-\alpha x_{i j}(t)+R \\
\xi_{i j}(t+1) & =\beta\left(F_{1}^{\boldsymbol{p}}(t)-F_{i j}^{\boldsymbol{p}}(t)\right) \\
x_{i j}(t+1) & =f\left(\zeta_{i j}(t+1)+\xi_{i j}(t+1)\right)
\end{aligned}
$$




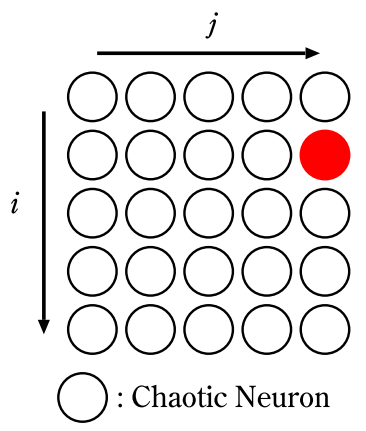

(a)

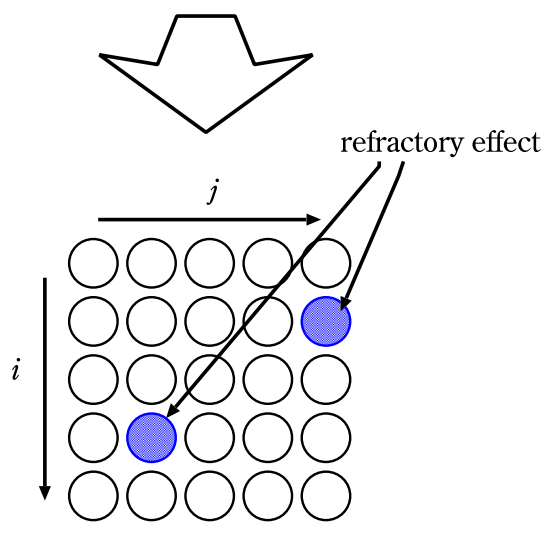

(c)

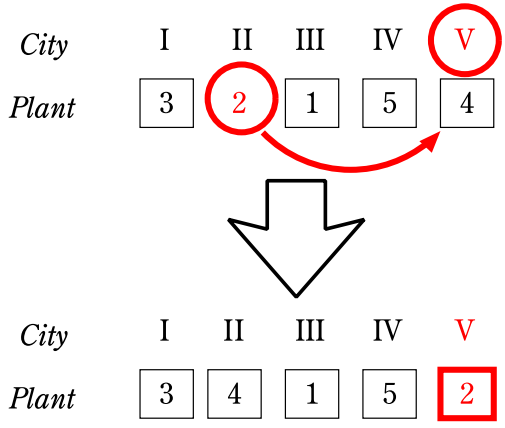

(b)

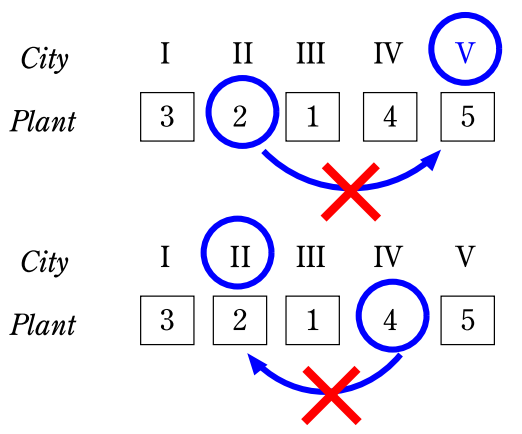

(d)

Fig. 4. The 2-opt method driven by chaotic neuro-dynamics for a size-5 QAP: (a) the $(2,5)$ th neuron is updated, and fires; (b) the 2-opt exchange assigns plant 2 to city $\mathrm{V} ;(\mathrm{c})$ refractory effects on the $(2,5)$ th neuron and $(4,2)$ th neuron; (d) the 2-opt exchanges that assign plant 2 to city $\mathrm{V}$ and plant 4 to city II are prohibited for a while because of the refractoriness.

where $\zeta_{i j}(t+1)$ corresponds to the tabu effect for the assignment of plant $i$ to city $j$, and $\xi_{i j}(t+1)$ gives the gain resulting from the assignment. $k_{r}$ is a decay parameter of the refractory effect $\left(0<k_{r}<1\right)$, $\alpha$ is a scaling parameter of the refractory effect $(\alpha>0), R$ is an external bias, and $f(\cdot)$ is a sigmoidal output function of the neuron. In addition, $F_{1}^{\boldsymbol{p}}(t)$ represents the current cost at time $t$, and $F_{i j}(t)$ is the cost after the DA method which initially doubly assigns plant $i$ to city $j$ in Step $\mathbf{1}$. When we asynchronously update the $(i, j)$ th neuron, we first calculate $\zeta_{i j}(t+1)$ and $\xi_{i j}(t+1)$ by Eqs. (7) and (8), respectively. In order to obtain $\xi_{i j}(t+1)$, we temporarily execute the DA method, which initially assigns plant $i$ to city $j$ in Step $\mathbf{1}$, to evaluate $F_{i j}^{\boldsymbol{p}}(t)$. We denote the resulting temporal solution as $\boldsymbol{p}_{\text {temp }}$. Then, if the $(i, j)$ th neuron fires, that is, $x_{i j}(t+1)>\frac{1}{2}$, we accept $\boldsymbol{p}_{\text {temp }}$ as a new solution, and proceed to update the next neuron. Otherwise, we discard $\boldsymbol{p}_{\text {temp }}$, and proceed to the next neuron.

We used numerical simulations to compare the performance of the exponential chaotic tabu search with the DA method with the original exponential chaotic tabu search, which is the evaluation standard in this paper as mentioned at the end of Section 4.1. In the simulations, we executed 30 trials with each method, and the number of exchanges in the permutation is fixed at $100 n$ in each trial for fair comparisons [32], where $n$ is the size of the problem. The left two columns, CS-2opt and CS-DA, of Table II summarize the simulation results for the exponential chaotic tabu searches with the 2-opt method and with the DA method, respectively. The results were obtained with Intel Core2 Quad CPU Q9650@ $3.00 \mathrm{GHz}$ with 3.6 GB of memory. The OS was Linux 2.6.27-67vl5, and the compiler was gcc++ with -O3 option. As shown in Table II, the exponential chaotic tabu search with the DA method resulted in better average gaps from the optimum or known-best solution than that with the 2-opt method, in 9 instances out of 15 . In contrast, however, the exponential chaotic tabu search with the DA method consumed much longer CPU-time than that with the 2-opt method for all instances. 
Table II. Comparisons among exponential chaotic tabu searches. The average gap from the optimum or known-best solution (AG (\%)), the success rate in finding the optimum or known-best solution (SR (\%)), and the CPUtime (CPU (sec.)) through 30 numerical simulation trials using the original exponential chaotic tabu search with the 2-opt method (CS-2opt), the exponential chaotic tabu search with the DA method (CS-DA), and the exponential chaotic tabu search with the MDA method (CS-MDA). Each run is cut at $100 n$ exchanges of the permutation, where $n$ is the size of the problem. The best result and second best for each problem are shown with bold and italic numbers, respectively.

\begin{tabular}{c|ccc|ccc|ccc}
\hline & \multicolumn{3}{|c|}{ CS-2opt } & \multicolumn{3}{c|}{ CS-DA } & \multicolumn{3}{c}{ CS-MDA } \\
\hline Instance & AG & SR & CPU & AG & SR & CPU & AG & SR & CPU \\
\hline Chr20a & 14.91 & 0 & 0.571 & 13.37 & 0 & 3.000 & $\mathbf{1 1 . 2 2}$ & $\mathbf{3 . 3 3}$ & $\mathbf{0 . 1 3 8}$ \\
Chr20b & 14.32 & 0 & 0.584 & 9.460 & 0 & 2.501 & $\mathbf{8 . 6 3 1}$ & 0 & $\mathbf{0 . 1 2 4}$ \\
Chr20c & 20.73 & 0 & 0.576 & $\mathbf{7 . 7 5 7}$ & 0 & 2.277 & 19.30 & 0 & $\mathbf{0 . 1 1 8}$ \\
Nug20 & 0.566 & 13.3 & 0.655 & 1.639 & 0 & 5.128 & $\mathbf{0 . 4 5 1}$ & $\mathbf{2 6 . 7}$ & $\mathbf{0 . 3 0 3}$ \\
Nug30 & 0.932 & 0 & $\mathbf{1 . 0 9 3}$ & 3.091 & 0 & 33.45 & $\mathbf{0 . 4 9 9}$ & $\mathbf{3 . 3 3}$ & 12.84 \\
Had20 & 0.251 & 0 & 0.503 & 0.679 & 0 & 1.624 & $\mathbf{0 . 2 3 4}$ & $\mathbf{1 3 . 3}$ & $\mathbf{0 . 1 5 1}$ \\
Rou20 & 2.358 & 0 & 1.575 & 3.356 & 0 & 1.683 & $\mathbf{1 . 2 0 3}$ & $\mathbf{3 . 3 3}$ & $\mathbf{0 . 2 0 0}$ \\
Scr20 & 6.941 & 0 & 0.421 & 4.819 & 0 & 2.715 & $\mathbf{0 . 9 8 7}$ & $\mathbf{2 6 . 7}$ & $\mathbf{0 . 1 4 8}$ \\
Sko42 & 1.971 & 0 & $\mathbf{6 . 5 7 7}$ & 4.421 & 0 & 62.45 & $\mathbf{0 . 5 1 4}$ & 0 & 19.33 \\
Tai20a & 3.345 & 0 & 1.629 & 2.303 & 0 & 7.339 & $\mathbf{2 . 0 9 1}$ & 0 & $\mathbf{0 . 2 7 8}$ \\
Tai30a & 4.584 & 0 & $\mathbf{0 . 1 0 2}$ & 4.399 & 0 & 55.21 & $\mathbf{1 . 5 5 2}$ & 0 & 27.70 \\
Tai40a & 2.364 & 0 & $\mathbf{1 2 . 3 8}$ & 5.478 & 0 & 233.3 & $\mathbf{1 . 9 5 1}$ & 0 & 44.37 \\
Tai20b & 5.644 & 0 & 0.391 & $\mathbf{2 . 0 4 2}$ & 0 & 1.087 & 3.133 & 0 & $\mathbf{0 . 1 2 1}$ \\
Tai30b & 4.360 & 0 & $\mathbf{0 . 5 2 6}$ & $\mathbf{2 . 1 8 8}$ & 0 & 6.657 & 2.622 & 0 & 1.155 \\
Tai40b & 5.135 & 0 & $\mathbf{1 . 1 7 8}$ & 2.702 & 0 & 18.39 & $\mathbf{2 . 5 6 1}$ & 0 & 3.334 \\
\hline
\end{tabular}

The DA method calculates three cost changes, $\Delta F_{i j}^{1}, \Delta F_{i j}^{2}$, and $\Delta F_{i j}^{3}$, given by Eqs. (4), (5), and (6), respectively. Moreover, the calculations for these cost changes take a large amount of time. This would be one of the reasons for the slow execution of the exponential chaotic tabu search with the DA method. In summary, although the DA method is superior to the 2-opt method as a local search as demonstrated in Table I, the introduction of chaotic neuro-dynamics into the DA method did not satisfactorily improve the performance of the DA method. Therefore, we modify the DA method in such a way that the chaotic search dynamics more effectively control the DA mechanism, in the next subsection.

\subsection{The exponential chaotic search with the modified double-assignment method}

In the original exponential chaotic tabu search with the 2-opt method mentioned in Section 4.1, firing of the corresponding neuron directly specifies the candidate for the 2-opt exchange; thus, chaotic neuro-dynamics completely control the 2-opt exchanges in the exponential chaotic tabu search. In contrast, in the exponential chaotic tabu search with the DA method, only the assignment in Step 1 is determined by the firing of a chaotic neuron. Therefore, chaotic neuro-dynamics do not completely control the double assignments. This may be the reason for the poor improvements indicated by Table II. We therefore propose the exponential chaotic tabu search with a modified DA (MDA) method, which enhances the effect of Step 1.

To that end, we impose a maximum value for $m$ to restrict the number of executions of Step 2 and Step 3 in the MDA. In practice, we set the maximum $m$ to 5 for a size-20 QAP, and 10 for size-30 and size-42 QAPs. The performance of the DA method itself, however, may be affected by the limitation on $m$. We therefore first evaluate the performance of the MDA method with limited $m$ as a local search. The results from the simulations of the MDA method are shown in the right column (MDA) of Table I. As shown in the table, the MDA method results in much better solutions than the 2-opt method for all problems, and has some advantages in the overall performance over the DA method. However, the MDA and DA methods are mostly comparable to each other as local searches. 
We finally evaluate the performance of the exponential chaotic tabu search with the MDA method through numerical simulations as shown in the right column (CS-MDA) of Table II. The table shows that the exponential chaotic tabu search with the MDA method resulted in the best, or at least the second best, average gaps from the optimum or known-best solutions for all instances. Moreover, the success rates in obtaining the optimum or known-best solutions of the exponential chaotic tabu search with the MDA method gave the best results. In terms of the CPU-time, the exponential chaotic tabu search with the MDA method is comparable to that with the 2-opt method. In fact, the exponential chaotic tabu search with the MDA method was quickest in 9 instances out of 15 . These results confirm that the limitation in $m$ in the MDA not only enhances the effect of chaotic neuro-dynamics improving the quality of solutions, but also contributes to the short CPU-time. As a consequence, we can conclude that the exponential chaotic tabu search with the MDA method has the best overall performance.

\section{Conclusions}

We first proposed the double-assignment (DA) method for QAPs based on the LK algorithm, which finds suboptimal solutions through temporary infeasible solutions, as a local search suitable for the exponential chaotic tabu search. In addition, we have shown that feature (F-2) of the LK algorithm for TSPs is also effective in solving QAPs. Second, we introduced chaotic neuro-dynamics to the DA method to implement the exponential decaying tabu effect and dynamical search to realize the exponential chaotic tabu search with the DA method. Furthermore, we imposed a maximum on $m$ in the DA method (MDA) in order to enhance the effect of chaotic neuro-dynamics on the DA mechanism. Finally, we proposed the exponential chaotic tabu search with the MDA method, and further, we confirmed that the proposed MDA method has excellent performance in solving QAPs when it is used in the exponential chaotic tabu search.

In future studies, we will improve the proposed method by also considering feature (F-1) of the LK algorithm. Furthermore, we will propose an automatic tuning method to find an appropriate maximum value for $m$. In addition, we will incorporate synchronous updates of the neuron states in the proposed method for massively parallel hardware implementations with an analog circuitry to drastically accelerate the execution time especially for large-size QAPs $[44,45]$.

\section{Acknowledgments}

This research is partially supported by the Aihara Innovative Mathematical Modelling Project, the Japan Society for the Promotion of Science (JSPS) through the "Funding Program for World-Leading Innovative R\&D on Science and Technology (FIRST Program)," initiated by the Council for Science and Technology Policy (CSTP). This work is also supported by Kakenhi (20300085).

\section{References}

[1] C.A. Floudas and P.M. Pardalos, Encyclopedia of Optimization, 2nd-edition, Springer-Verlag, 2008.

[2] G.A. Croes, "A method for solving traveling salesman problems," Operations Research, vol. 6, pp. $791-812,1958$.

[3] B. Chandra, H. Karloff, and C. Tovey, "New results on the old k-opt algorithm for the TSP," SIAM Journal on Computing, vol. 28, no. 6, pp. 150-159, 1999.

[4] I. Or, "Traveling salesman-type combinatorial problems and their relation to the logistics of regional blood banking," Ph.D. Thesis, Department of Industrial Engineering and Management Science, Northwestern University, Evanston, Illinois, 1967.

[5] S. Lin and B. Kernighan, "An effective heuristic algorithm for the traveling salesman problem," Operations Research, vol. 21, pp. 498-516, 1973.

[6] F. Glover, "New ejection chain and alternation path methods for traveling salesman problems," Computer Science and Operations Research, pp. 449-509, 1992.

[7] S. Kirkpatrick, C.D. Gelatt, and M.P. Vecchi, "Optimization by simulated annealing," Science, vol. 220, pp. 671-680, 1983. 
[8] C. Fleurent and J.A. Ferland, "Genetic hybrids for the quadratic assignment problem," DIMACS Series in Mathematics and Theoretical Computer Science, vol. 16, pp. 173-187, 1994.

[9] F. Glover, "Tabu Search - Part I," ORSA Journal on Computing, vol. 1, pp. 190-206, 1989.

[10] R. Battiti and G. Tecchiolli, "The reactive tabu search," ORSA Journal on Computing, vol. 6, pp. 126-140, 1994.

[11] E.D. Taillard, "Robust taboo search for the quadratic assignment problem," Parallel Computing, vol. 17, pp. 443-455, 1991.

[12] M. Hanan and J.M. Kurtzberg, "A review of the placement problems and quadratic assignment problems," SIAM Review, vol. 14, no. 2, pp. 324-342, 1972.

[13] G. Finke, R.E. Burkard, and F. Rendl, "Quadratic assignment problems," Annals of Discrete Mathematics, vol. 31, pp. 61-82, 1987.

[14] S. Sahni and T. Gonzalez, "P-complete approximation problems," Journal of the ACM, vol. 23, pp. 555-565, 1976.

[15] F. Hillier and M. Michael, "Quadratic assignment problem algorithms and the location of indivisible facilities," Management Science, vol. 13, no. 1, pp. 42-57, 1966.

[16] R.E. Brekard, S.E. Karisch, and R. Rendl, "QAPLIB - A quadratic assignment problem library," http://www.seas.upenn.edu/qaplib/

[17] K.A. Murthy, P. Pardalos, and Y. Li, "A local search algorithm for the quadratic assignment problem," Informatica, vol. 3, pp. 524-538, 1992.

[18] E.D. Taillard, "Comparison of iterative searches for the quadratic assignment problem," Location Science, vol. 3, no. 2, pp. 87-105, 1995.

[19] K. Aihara, T. Takabe, and M. Toyoda, "Chaotic neural networks," Phys. Lett. A, vol. 144, pp. 333-340, 1990.

[20] M. Adachi and K. Aihara, "Associative dynamics in a chaotic neural network," Neural Networks, vol. 10, no. 1, pp. 83-98, 1997.

[21] K. Aihara, "Chaos engineering and its application to parallel distributed processing with chaotic neural networks," Proc. of the IEEE, vol. 90, no. 5, pp. 919-930, 2002.

[22] Y. Hayakawa, A. Marumoto, and Y. Sawada, "Effects of the chaotic noise on the performance of a neural network model for optimization problems," Phys. Rev. E, vol. 51, pp. 2693-2696, 1995.

[23] M. Hasegawa, T. Ikeguchi, T. Matozaki, and K. Aihara, "Analysis of additive effects of nonlinear dynamics for combinatorial optimization," IEICE Trans. Fundamentals, vol. E80-A, no. 1, pp. 206-213, 1997.

[24] M. Hasegawa and T. Ikeguchi, "Combination of chaotic neuro-dynamics with the 2-opt algorithm to solve traveling salesman problems," Phys. Rev. Lett., vol. 79, no. 12, pp. 2344-2347, 1977.

[25] M. Hasegawa, T. Ikeguchi, and K. Aihara, "Solving large scale traveling salesman problems by chaotic neuro-dynamics," Neural Networks, vol. 15, pp. 271-283, 2002.

[26] M. Hasegawa, T. Ikeguchi, and K. Aihara, "On the effect of the $k$-opt method with chaotic neurodynamics," IEICE Technical Report, vol. 101, NLP2001-37, pp. 25-32, 2001.

[27] T. Matsuura and T. Ikeguchi, "Chaotic search for traveling salesman problems by using 2-opt and Or-opt algorithms," Lecture Note in Computer Science, vol. 5164, pp. 587-596, 2008.

[28] S. Motohashi, T. Matsuura, and T. Ikeguchi, "Chaotic search method using the Lin-Kernighan algorithm for traveling salesman problems," Proc. NOLTA'08, pp. 144-147, 2008.

[29] S. Motohashi, T. Matsuura, T. Ikeguchi, and K. Aihara, "The Lin-Kernighan algorithm driven by chaotic neuro-dynamics for large scale traveling salesman problems," Lecture Note in Computer Science, vol. 5769, pp. 563-572, 2009.

[30] S. Motohashi, T. Matsuura, and T. Ikeguchi, "Chaotic search based on the ejection chain method for traveling salesman problems," Proc. NOLTA'09, pp. 304-307, 2009.

[31] M. Hasegawa, T. Ikeguchi, and K. Aihara, "Exponential and chaotic neurodynamical tabu searches for quadratic assignment problems," Control and Cybernetics, vol. 29, pp. 773-788, 2000 .

[32] M. Hasegawa, T. Ikeguchi, and K. Aihara, "A novel chaotic search for quadratic assignment 
problems," European Journal of Operational Research, vol. 139, pp. 543-556, 2002.

[33] T. Matsuura and T. Ikeguchi, "Chaotic motif sampler: Detecting motifs from biological sequences by using chaotic neurodynamics," Nonlinear Theory and Its Applications, IEICE, vol. 1, no. 1, pp. 207-220, 2010.

[34] T. Hoshino, T. Kimura, and T. Ikeguchi, "Two simple local searches controlled by chaotic dynamics for vehicle routing problems with time window," Proc. of Metaheuristics Int. Conf., CD-ROM, 2007.

[35] T. Kimura and T. Ikeguchi, "An optimum strategy for dynamic and stochastic packet routing problems by chaotic neuro-dynamics," Integrated Computer-Aided Engineering, vol. 14, no. 4, pp. 307-322, 2007.

[36] K. Kaneko and I. Tsuda, "Chaotic itinerancy," Chaos, vol. 13, no. 3, pp. 926-936, 2003.

[37] L. Blum, M. Shub, and S. Smale, "On a theory of computation and complexity over the real numbers: NP-completeness, recursive functions and universal machines," Bull. Am. Math. Soc., vol. 21, no. 1, pp. 1-46, 1989.

[38] L. Blum, F. Cucker, M. Shub, and S. Smale, Complexity and Real Computation, Springer-Verlag, New York, NY, 1997.

[39] H.T. Siegelmann, A. Ben-Hur, and S. Fishman, "Computational complexity for continuous time dynamics," Phys. Rev. Lett., vol. 83, no. 7, pp. 1463-1466, 1999.

[40] Y. Horio, T. Ikeguchi, and K. Aihara, "A mixed analog/digital chaotic neuro-computer system for quadratic assignment problems," Neural Networks, vol. 18, no. 5, pp. 503-513, 2005.

[41] Y. Horio and K. Aihara, "Analog computation through high-dimensional physical chaotic neurodynamics," Physica D: Nonlinear Phenomena, vol. 237, no. 9, pp. 1215-1225, 2008.

[42] K. Tanaka, Y. Horio, and K. Aihara, "A modified algorithm for the quadratic assignment problem using chaotic-neuro-dynamics for VLSI implementation," Proc. Int. Joint Conf. on Neural Networks, vol. 1, pp. 240-245, 2001.

[43] Y. Kobayashi, T. Koyama, S. Matsui, Y. Horio, and K. Aihara, "Exponential chaotic tabu search hardware for quadratic assignment problems using switched-current chaotic neuron IC," Proc. Int. Joint Conf. on Neural Networks, vol. 3, pp. 2221-2225, 2004.

[44] T. Kawamura, Y. Horio, and M. Hasegawa, "Mutual information analysis of chaotic neurodynamics driven by neuron selection methods in synchronous exponential chaotic tabu search for quadratic assignment problems," Lecture Notes in Computer Science, vol. 6433, no. 1, Springer, pp. 49-57, 2010.

[45] N. Yokota, Y. Horio, K. Aihara, and M. Hasegawa, "A synchronous exponential chaotic tabu search for quadratic assignment problems," IEICE Technical Report, vol. 107, pp. 49-54, 2008 (in Japanese).

[46] K. Shibata and Y. Horio, "An application of Lin-Kernighan algorithm to quadratic assignment problems," IEICE Technical Report, vol. 109, NLP2009-164, pp. 37-42, 2010 (in Japanese).

[47] E.S. Buffa, G.C. Armour, and T.E. Vollmann, "Allocating facilities with CRAFT," Harvard Business Review, pp. 136-158, March 1964.

[48] G. Reinelt, "TSPLIB - A traveling salesman problem library," http://www2.iwr.uni-heidelberg.de/groups/comopt/software/TSPLIB95/ 